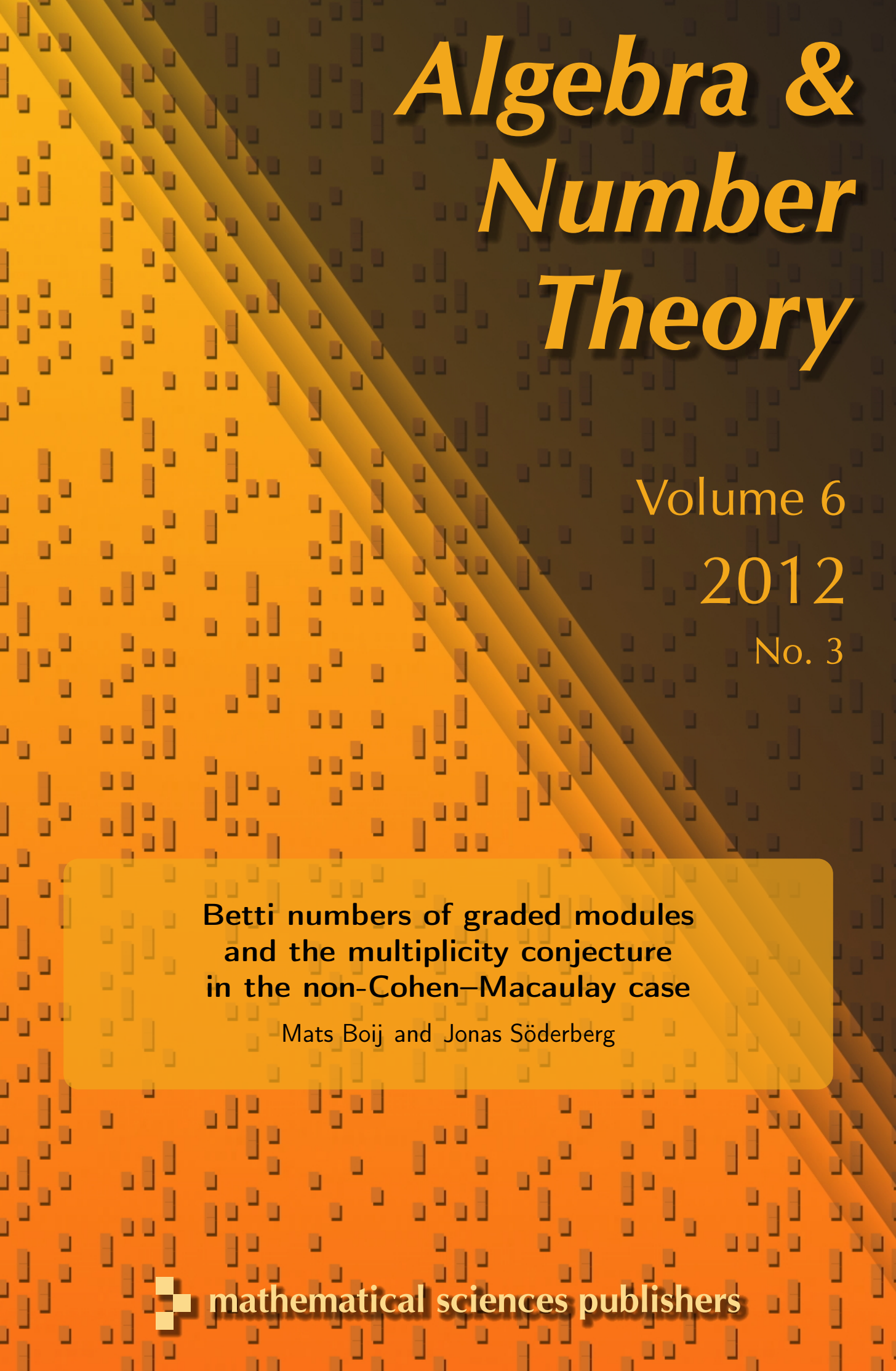

Betti numbers of graded modules and the multiplicity conjecture in the non-Cohen-Macaulay case

-Mats Boij and Jonas Söderberg

\lrcorner 


\title{
Betti numbers of graded modules and the multiplicity conjecture in the non-Cohen-Macaulay case
}

\author{
Mats Boij and Jonas Söderberg
}

\begin{abstract}
We use results of Eisenbud and Schreyer to prove that any Betti diagram of a graded module over a standard graded polynomial ring is a positive linear combination of Betti diagrams of modules with a pure resolution. This implies the multiplicity conjecture of Herzog, Huneke, and Srinivasan for modules that are not necessarily Cohen-Macaulay and also implies a generalized version of these inequalities. We also give a combinatorial proof of the convexity of the simplicial fan spanned by pure diagrams.
\end{abstract}

\section{Introduction}

The formula for the multiplicity of a standard graded algebra with a pure resolution found by C. Huneke and M. Miller [Huneke and Miller 1985], led to the formulation of the multiplicity conjecture by Huneke and H. Srinivasan, which was later generalized and published by J. Herzog and Srinivasan [Herzog and Srinivasan 1998]. J. Migliore, U. Nagel and T. Römer [Migliore et al. 2008a] extended the conjecture to modules and strengthened it.

In a series of papers [Srinivasan 1998; Gold 2003; Guardo and Van Tuyl 2005; Migliore et al. 2005; 2008b; Römer 2005; Francisco 2006; Herzog and Zheng 2006; Kubitzke and Welker 2006; Miró-Roig 2006; Francisco and Van Tuyl 2007; Gold et al. 2007; Seo and Srinivasan 2007; Zanello 2007; Goff 2008; Hibi and Singla 2008; Puthenpurakal 2008] different versions of the multiplicity conjecture have been proven in many special cases. An exposition of most of these results can be found in [Francisco and Srinivasan 2007].

Recently D. Eisenbud and F.-O. Schreyer [2009] proved the strongest version of the conjecture for Cohen-Macaulay modules by proving a set of conjectures formulated in [Boij and Söderberg 2008] on the set of possible Betti diagrams up to multiplication by positive rational numbers. Eisenbud and Schreyer introduced a set of linear functionals defined on the space of possible Betti diagrams. The

MSC2000: primary 13D02; secondary $13 \mathrm{~A} 02$.

Keywords: graded modules, Betti numbers, multiplicity conjecture. 
linear functionals are given by certain cohomology tables of vector bundles on $\mathbb{P}^{n-1}$, and they showed that the supporting hyperplanes of the exterior facets of the simplicial fan given by the pure Betti diagrams are given by the vanishing of these linear functionals, while the functionals are nonnegative on the Betti diagram of any minimal free resolution.

In this paper we generalize the construction given in [Boij and Söderberg 2008] to include Cohen-Macaulay pure Betti diagrams of various codimensions. We show that linear functionals similar to those introduced by Eisenbud and Schreyer define the supporting hyperplanes of the simplicial fan. Furthermore, these new linear functionals are limits of the Eisenbud-Schreyer functionals, which allows us to conclude that all Betti diagrams of graded modules can be uniquely written as positive linear combinations of pure diagrams in totally ordered chains. Together with the existence of modules with pure resolutions, proved by D. Eisenbud, G. Fløystad and J. Weyman [Eisenbud et al. 2011] in characteristic zero and by Eisenbud and Schreyer [Eisenbud and Schreyer 2009] in general, this gives a complete classification of the possible Betti diagrams up to multiplication by scalars.

As a consequence, we get the multiplicity conjecture for algebras and modules that are not necessarily Cohen-Macaulay. In fact, we get a stronger version of the inequalities of the multiplicity conjecture in terms of the Hilbert series of the module which is bounded from below by the Hilbert series corresponding to the lowest shifts in a minimal free resolution while it is bounded from above by the Hilbert series corresponding to the highest shifts in the first $s+1$ terms of the resolution, where $s$ is the codimension of the module.

We also give a combinatorial proof of the convexity of the simplicial fan spanned by the pure diagrams, even though this convexity is an implicit consequence of the results involving the linear functionals.

Furthermore, we show that if we choose the basis of pure diagrams in a certain way, all the coefficients in the expansion of an actual Betti diagram into a chain of pure diagrams are nonnegative integers.

Remark 1.1. Before the submission of this paper, Eisenbud and Schreyer [2010] have gone further on the side of cohomology tables and extended their results from vector bundles to coherent sheaves. Apart from the addition of two remarks, this manuscript is in its original form and is not dependent on these newer developments.

\section{The partially ordered set of pure Betti diagrams}

Let $R=k\left[x_{1}, x_{2}, \ldots, x_{n}\right]$ be the polynomial ring with the standard grading. For any finitely generated graded module $M$, we have a minimal free resolution

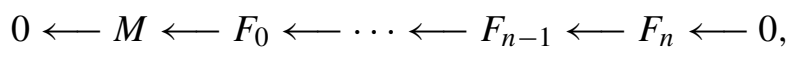


where

$$
F_{i}=\bigoplus_{j \in \mathbb{Z}} R(-j)^{\beta_{i, j}}, \quad i=0,1, \ldots, n
$$

and we get that the Hilbert series of $M$ can be recovered from the Betti numbers, $\beta_{i, j}$, by

$$
H(M, t)=\frac{1}{(1-t)^{n}} \sum_{i=0}^{n} \sum_{j \in \mathbb{Z}}(-1)^{i} \beta_{i, j} t^{j} .
$$

It was noted by Herzog and M. Kühl [Herzog and Kühl 1984] that this gives us $s$ linearly independent equations

$$
\sum_{i=0}^{n} \sum_{j \in \mathbb{Z}}(-1)^{i} \beta_{i, j} j^{m}=0, \quad m=0,1, \ldots, s-1,
$$

where $s$ is the codimension of $M$.

Furthermore, they proved that in the case when $M$ is Cohen-Macaulay and the resolution is pure, that is, if $F_{i}=R\left(-d_{i}\right)$ for some integers $d_{0}, d_{1}, \ldots, d_{s}$, we get a unique solution (up to scalar multiples) to these equations given by

$$
\beta_{i, j}= \begin{cases}(-1)^{i} \prod_{\substack{j=0 \\ j \neq i}}^{s} \frac{1}{d_{j}-d_{i}}, & j=d_{i}, \\ 0, & j \neq d_{i},\end{cases}
$$

for $i=0,1, \ldots, s$.

Definition 2.1. For an increasing sequence of integers $\boldsymbol{d}=\left(d_{0}, d_{1}, \ldots, d_{s}\right)$, where $0 \leq s \leq n$, we denote by $\pi(\boldsymbol{d})$ the matrix in $\mathbb{Q}^{n-1} \times \mathbb{Q}^{\mathbb{Z}}$ given by

$$
\pi(\boldsymbol{d})_{i, j}=(-1)^{i} \prod_{\substack{j=0 \\ j \neq i}}^{s} \frac{1}{d_{j}-d_{i}}, \quad \text { for } j=d_{i}
$$

and zero elsewhere. We will call this the pure diagram given by the degree sequence $\boldsymbol{d}=\left(d_{0}, d_{2}, \ldots, d_{s}\right)$. We will use the notation $d_{i}(\pi)$ to denote the degree, $d_{i}$, when $\pi=\pi(\mathbf{d})=\pi\left(d_{0}, d_{1}, \ldots, d_{s}\right)$.

For degree sequences with $d_{0}=0$, we will also use the normalized pure diagram

$$
\bar{\pi}(\boldsymbol{d})=d_{1} d_{2} \cdots d_{s} \pi(\boldsymbol{d})
$$

so that normalized pure diagrams have $\bar{\pi}_{0,0}=1$.

We define a partial ordering on the set of pure diagrams, extending the ordering used for Cohen-Macaulay diagrams of a fixed codimension. 
Definition 2.2. We say that $\pi\left(d_{0}, d_{1}, \ldots, d_{s}\right) \leq \pi\left(d_{0}^{\prime}, d_{1}^{\prime}, \ldots, d_{t}^{\prime}\right)$ if the $s \geq t$ and $d_{i} \leq d_{i}^{\prime}$ for $i=0,1, \ldots, t$.

As in the case of Cohen-Macaulay pure diagrams, we get a simplicial structure given by the maximal chains of pure diagrams, which spans simplicial cones. In order to have maximal chains in this setting, we have to fix a bound on the region we are considering. We can do this by restricting the degrees to be in a given region $M+i \leq d_{i} \leq N+i$. We will denote the subspace generated by Betti diagrams with these restrictions by $B_{M, N}=\mathbb{Q}^{n+1} \times \mathbb{Q}^{N-M+1}$. Furthermore, we denote by $B_{M, N}^{s}$ the subspace of $B_{M, N}$ of diagrams satisfying the $s$ first Herzog-Kühl equations.

Proposition 2.3. For $s=0,1, \ldots, n$, we have that any maximal chain of pure diagrams of codimension at least $s$ in $B_{M, N}$ form a basis for $B_{M, N}^{s}$.

Proof. For any interval of length one $\pi<\pi^{\prime}$ there is a unique nonzero entry in $\pi$ which is zero in all pure diagrams above $\pi^{\prime}$. Thus the pure diagrams in any maximal chain are linearly independent. The number of elements in any maximal chain of pure diagrams of codimension at least $s$ is $(n+1)(N-M)+n-s+1$, since we have $n+1$ positions that has to be raised $N-M$ steps and then $n-s+1$ times when the codimension is lowered by one. On the other hand, we have that the dimension of $B_{M, N}^{s}$ is $(n+1)(N-M+1)-s$, since we have $s$ independent equations on $B_{M, N}$.

By looking at the order in which the different positions of a Betti diagram in $B_{M, N}$ disappear when going along a maximal chain of pure diagrams we get the following observation:

Proposition 2.4. The maximal chains of pure diagrams in $B_{M, N}$ are in one to one correspondence with numberings of the entries of an $(N-M+1) \times(n+1)$-matrix which are increasing to the left and downwards.

Example 2.5. For $n=2, M=0$, and $N=1$, the numbering

\begin{tabular}{|l|l|l|}
\hline 5 & 3 & 1 \\
\hline 6 & 4 & 2 \\
\hline
\end{tabular}

corresponds to the maximal chain

$$
\begin{array}{rrr}
* & * & * \\
- & - & -
\end{array}<\begin{array}{lll}
* & * & - \\
- & - & *
\end{array}<<\begin{array}{lll}
* & * & - \\
- & - & -
\end{array}<<\begin{array}{lll}
* & - & - \\
- & * & -
\end{array}<<\begin{array}{lll}
* & - & - \\
- & - & -
\end{array}<<\begin{array}{lll}
- & - & - \\
* & - & - \\
\hline
\end{array}
$$

and there are four other maximal chains corresponding to

\begin{tabular}{|l|l|l|}
\hline 3 & 2 & 1 \\
\hline 6 & 5 & 4 \\
\hline
\end{tabular}

\begin{tabular}{|l|l|l|}
\hline 4 & 2 & 1 \\
\hline 6 & 5 & 3 \\
\hline
\end{tabular}

\begin{tabular}{|l|l|l|}
\hline 5 & 2 & 1 \\
\hline 6 & 4 & 3 \\
\hline
\end{tabular}

and

\begin{tabular}{|l|l|l|}
\hline 4 & 3 & 1 \\
\hline 6 & 5 & 2 \\
\hline
\end{tabular}




\section{Description of the boundary facets}

We know that the partially ordered set of pure diagrams in $B_{M, N}$ give rise to a simplicial fan $\Delta$, where the faces are the totally ordered subsets. The facets of this simplicial fan correspond to maximal chains in the partially ordered set. According to Proposition 2.3 each such set is a basis for the space $B_{M, N}$. If we look at normalized Betti diagrams, we get a simplicial complex which is the hyperplane section of the simplicial fan. This is the complex described in our previous paper, in the case of Cohen-Macaulay diagrams (see [Boij and Söderberg 2008, Proposition 2.9]).

Proposition 3.1. The simplicial cones spanned by the totally ordered sets of pure diagrams in $B_{M, N}$ form a simplicial fan.

Proof. We need to show that the cones meet only along faces. This is the same as to say that any element which can be written as a positive linear combination of pure diagrams in a chain can be written so in a unique way. If such a sum has only one term, the uniqueness is trivial. Thus, suppose that a diagram in $B_{M, N}$ can be written as a positive linear combination of totally ordered pure diagrams in two different ways and that this is the minimal number of terms in such an example. We then have

$$
\beta=\sum_{i=1}^{m} \lambda_{i} \pi_{i}=\sum_{j=1}^{k} \mu_{j} \pi_{j}^{\prime},
$$

where all the coefficients are positive. Look at the lowest degree in which $\beta$ is nonzero for each column. These degrees have to be given by the degrees in $\pi_{1}$ and by the degrees in $\pi_{1}^{\prime}$. Thus we must have $\pi_{1}=\pi_{1}^{\prime}$. If $\lambda_{1}>\mu_{1}$, we can subtract $\mu_{1} \pi_{1}$ from $\beta$ and from both sums to get

$$
\beta-\lambda_{1} \pi_{1}=\sum_{i=2}^{m} \lambda_{i} \pi_{i}=\left(\mu_{1}-\lambda_{1}\right) \pi_{1}+\sum_{j=2}^{k} \mu_{j} \pi_{j}^{\prime} .
$$

Since all the pure diagrams in the left expression are greater than $\pi_{1}$, the degrees in which $\beta-\lambda_{1} \pi_{1}$ is nonzero have to be given by $\pi_{2}$, but then the coefficient of $\pi_{1}$ in the right-hand expression has to be zero, that is, $\lambda_{1}=\mu_{1}$. Since this was assumed to be a minimal example, we get that the expressions for $\beta-\lambda_{1} \pi_{1}$ are term wise equal and so were the original expressions for the diagram $\beta$.

We will now show that the coefficients of the pure diagrams when a Betti diagram is expanded in the basis given by a maximal chain have nice expressions. In particular, we see that the coefficients are integers. Moreover, it will give us expressions for the inequalities that define the simplicial fan similar to the 
inequalities used by Eisenbud and Schreyer to prove our conjectures in the CohenMacaulay case.

Proposition 3.2. The coefficient of $\pi_{1}=\pi\left(d_{0}, d_{1}, \ldots, d_{m}\right)$ when a Betti diagram $\beta$ in $B_{M, N}^{s}$ is expanded in a basis containing $\pi_{0}<\pi_{1}<\pi_{2}$ is given by the following formulas:

(a) When $\pi_{1}$ differs from $\pi_{0}$ in codimension and from $\pi_{2}$ in column $k$, it is given by

$$
\sum_{i=0}^{n} \sum_{d=M}^{d_{i}\left(\pi_{0}\right)}(-1)^{i} \prod_{\substack{j=0 \\ j \neq k}}^{m}\left(d_{j}-d\right) \beta_{i, d} .
$$

(b) When $\pi_{1}$ differs from $\pi_{0}$ in codimension and from $\pi_{2}$ in column $k$, it is given by

$$
\sum_{i=0}^{n} \sum_{d=M}^{d_{i}\left(\pi_{0}\right)}(-1)^{i}\left(d_{k}-d_{m}\right) \prod_{\substack{j=0 \\ j \neq k}}^{m-1}\left(d_{j}-d\right) \beta_{i, d} .
$$

(c) When $\pi_{1}$ differs from $\pi_{2}$ in column $k$ and from $\pi_{0}$ in column $\ell \neq k$, it is given by

$$
\sum_{i=0}^{n} \sum_{d=M}^{d_{i}\left(\pi_{0}\right)}(-1)^{i}\left(d_{\ell}-d_{k}\right) \prod_{\substack{j=0 \\ j \notin\{k, \ell\}}}^{m}\left(d_{j}-d\right) \beta_{i, d} .
$$

(d) When $\pi_{1}$ differs from $\pi_{0}$ and $\pi_{2}$ in codimension, it is given by

$$
\sum_{i=0}^{n} \sum_{d=M}^{d_{i}\left(\pi_{0}\right)}(-1)^{i} \prod_{j=0}^{m-1}\left(d_{j}-d\right) \beta_{i, d}
$$

In particular, all the coefficients are integers.

Remark 3.3. For simplicity of notation, we use the convention that $d_{i}(\pi)=N$ if $i$ is greater than the codimension of $\pi$.

Observe that when $\pi_{1}$ differs from $\pi_{0}$ and $\pi_{2}$ in column $k$, the coefficient is given by the individual Betti number $(-1)^{k} \prod_{j \neq k}\left(d_{j}-d_{k}\right) \beta_{k, d_{k}}$, since no other diagram than $\pi_{1}$ contributes in this position, $\left(k, d_{k}\right)$.

Proof. In all four cases, (a)-(d), the coefficients of $\beta_{i, d}$ are zero for pure diagrams $\pi \geq \pi_{2}$ by construction since the sums are taken only up to degree $d_{i}\left(\pi_{0}\right)$ and the coefficients are zero in all positions where $d_{i}\left(\pi_{0}\right)=d_{i}\left(\pi_{2}\right)$.

Furthermore, the expressions are zero on all pure diagrams $\pi$, where $\pi \leq \pi_{0}$ by the Herzog-Kühl equations. Indeed, for such pure diagrams, we can extend the summation over $d$ to be over all degrees from $M$ to $N$. When we do this and 
expand the products we get polynomials in $d$ and, therefore, the expressions are linear combinations of the Herzog-Kühl equations

$$
\sum_{i=0}^{n} \sum_{d=M}^{N}(-1)^{i} \beta_{i, d} d^{j}=0
$$

for $j=0,1, \ldots, m-1$ in cases (b) and (d), and for $j=0,1, \ldots, m$ in cases (a) and (d). Observe that all pure diagrams $\pi$ with $\pi \leq \pi_{0}$, have codimension at least the codimension of $\pi_{0}$ and hence satisfy these Herzog-Kühl equations.

It remains to show that the value of the expressions are one on the pure diagram $\pi_{1}$. In case (a), the only nonzero term is in column $k$ and equals

$$
(-1)^{k} \prod_{\substack{j=0 \\ j \neq k}}^{m}\left(d_{j}-d_{k}\right) \cdot(-1)^{k} \prod_{\substack{j=0 \\ j \neq k}}^{m} \frac{1}{d_{j}-d_{k}}=1
$$

in case (b), the only nonzero term is in column $m$ and equals

$$
(-1)^{m}\left(d_{k}-d_{m}\right) \prod_{\substack{j=0 \\ j \neq k}}^{m}\left(d_{j}-d_{m}\right) \cdot(-1)^{m} \prod_{j=0}^{m-1} \frac{1}{d_{j}-d_{m}}=1
$$

in case (c), the only nonzero term is in column $k$ and equals

$$
(-1)^{k}\left(d_{\ell}-d_{k}\right) \prod_{\substack{j=0 \\ j \notin\{k, \ell\}}}^{m}\left(d_{j}-d_{k}\right) \cdot(-1)^{k} \prod_{\substack{j=0 \\ j \neq \ell}}^{m} \frac{1}{d_{j}-d_{k}}=1,
$$

and in case (d), the nonzero term is in column $m$ and equals

$$
(-1)^{m} \prod_{j=0}^{m-1}\left(d_{j}-d_{m}\right) \cdot(-1)^{m} \prod_{j=0}^{m-1} \frac{1}{d_{j}-d_{m}}=1 .
$$

Example 3.4. When $n=3, M=0$, and $N=2$ we can choose the basis of $B_{0,2}$ given by the numbering

\begin{tabular}{|l|l|l|l|}
\hline 10 & 4 & 3 & 1 \\
\hline 11 & 6 & 5 & 2 \\
\hline 12 & 9 & 8 & 7 \\
\hline
\end{tabular}


When expanding a Betti diagram

\begin{tabular}{c|cccc} 
& $\beta_{0}$ & $\beta_{1}$ & $\beta_{2}$ & $\beta_{3}$ \\
\hline $0:$ & $\beta_{0,0}$ & $\beta_{1,1}$ & $\beta_{2,2}$ & $\beta_{3,3}$ \\
$1:$ & $\beta_{0,1}$ & $\beta_{1,2}$ & $\beta_{2,3}$ & $\beta_{3,4}$ \\
$2:$ & $\beta_{0,2}$ & $\beta_{1,3}$ & $\beta_{2,4}$ & $\beta_{3,5}$
\end{tabular}

(where we use the Macaulay2 [Grayson and Stillman 1993] convention shifting the columns upwards in order for the diagrams to be more compact) into this chain we get the coefficients by applying the linear functionals corresponding to the following matrices:

\begin{tabular}{|llll|}
\hline 0 & 0 & 0 & 6 \\
0 & 0 & 0 & 0 \\
0 & 0 & 0 & 0 \\
\hline
\end{tabular}

1

\begin{tabular}{|rrrr|}
\hline 0 & 8 & -12 & 12 \\
0 & 0 & 0 & 8 \\
0 & 0 & 0 & 0 \\
\hline
\end{tabular}

4

\begin{tabular}{|rrrr|}
\hline 0 & -6 & 8 & -6 \\
0 & -8 & 6 & 0 \\
0 & 0 & 0 & 10 \\
\hline
\end{tabular}

7

\begin{tabular}{|llll|}
\hline 1 & -1 & 1 & -1 \\
0 & -1 & 1 & -1 \\
0 & -1 & 1 & -1 \\
\hline
\end{tabular}

10

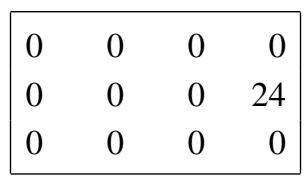

2

\begin{tabular}{|rrrr|}
\hline 0 & -4 & 6 & -6 \\
0 & 0 & 6 & 4 \\
0 & 0 & 0 & 0 \\
\hline
\end{tabular}

5

\begin{tabular}{|rrrr|}
\hline 0 & 2 & -2 & 0 \\
0 & 2 & 0 & -4 \\
0 & 0 & 4 & -10 \\
\hline
\end{tabular}

8

\begin{tabular}{|llll|}
\hline 0 & 0 & 0 & 0 \\
1 & 0 & 0 & 0 \\
0 & 0 & 0 & 0 \\
\hline
\end{tabular}

11

\begin{tabular}{|c|c|c|}
\hline 0 & 0 & $6-18$ \\
\hline 0 & 0 & $0-36$ \\
\hline 0 & 0 & 0 \\
\hline
\end{tabular}

3

\begin{tabular}{|rrrr}
0 & 8 & -12 & 12 \\
0 & 12 & -12 & 8 \\
0 & 0 & 0 & 0 \\
\hline
\end{tabular}

6

\begin{tabular}{|llll|}
\hline 0 & 1 & -2 & 3 \\
0 & 2 & -3 & 4 \\
0 & 3 & -4 & 5 \\
\hline
\end{tabular}

9

\begin{tabular}{|llll|}
\hline 0 & 0 & 0 & 0 \\
0 & 0 & 0 & 0 \\
1 & 0 & 0 & 0 \\
\hline
\end{tabular}

12

Matrices 1, 2, 11, and 12 correspond to individual Betti numbers as we will see in the next proposition. Matrices 3, 4, 5, and 6 are as in Proposition 3.2 case (c), since they correspond to two consecutive degree changes in distinct columns. Matrix 7 is as in case (b), since it corresponds to a degree change followed by a change in codimension. Matrices 8 and 9 are as in case (d), since they correspond to two consecutive changes of codimension. Matrix 10 is as in case (a) since it corresponds to a change in codimension followed by a degree change. 
In order to prove that the simplicial fan is convex and in order to prove that any Betti diagram of a module of codimension $s$ is a positive linear combination of pure diagrams, we need to know the boundary of the simplicial fan. The description is very similar to the description given in our previous paper [Boij and Söderberg 2008, Proposition 2.12] and we only have to add one more kind of boundary facet.

Proposition 3.5. A facet of the boundary of the simplicial fan given by the pure diagrams in $B_{M, N}^{s}$ is given by removing one element from a maximal chain such that there is a unique way to complete it to a maximal chain. There are four different cases:

(i) The removed element is maximal or minimal.

(ii) The removed element is in the middle of a chain of three degree sequences which differ in one single column, that is,

$$
\pi(\boldsymbol{d})<\pi\left(\boldsymbol{d}^{\prime}\right)<\pi\left(\boldsymbol{d}^{\prime \prime}\right), \quad d_{i}=d_{i}^{\prime}-1=d_{i}^{\prime \prime}-2 .
$$

(iii) The removed element differs from the adjacent vertices in two adjacent degrees, that is,

$$
\pi(\boldsymbol{d})<\pi\left(\boldsymbol{d}^{\prime}\right)<\pi\left(\boldsymbol{d}^{\prime \prime}\right), \quad d_{i+1}=d_{i+1}^{\prime}-1, \quad d_{i}^{\prime}=d_{i}^{\prime \prime}-1 .
$$

(iv) the removed element differs from the adjacent vertices in codimension, that is,

$$
\pi(\boldsymbol{d})<\pi\left(\boldsymbol{d}^{\prime}\right)<\pi\left(\boldsymbol{d}^{\prime \prime}\right), \quad \operatorname{codim}(\pi(\boldsymbol{d}))=\operatorname{codim}\left(\pi\left(\boldsymbol{d}^{\prime \prime}\right)\right)+2 .
$$

Proof. A boundary facet is a codimension-one face of a simplicial cone and hence given by the removing of one vertex. Since it is on the boundary, it is contained in a unique cone of maximal dimension, so there has to be a unique extension of the chain into a maximal chain.

This clearly happens if we remove the maximal or the minimal element, since there are unique such elements in the partially ordered set. Suppose therefore that we remove $\pi\left(\boldsymbol{d}^{\prime}\right)$ and that the adjacent vertices in the chain are $\pi(\boldsymbol{d})<\pi\left(\boldsymbol{d}^{\prime}\right)$ and $\pi\left(\boldsymbol{d}^{\prime \prime}\right)>\pi\left(\boldsymbol{d}^{\prime}\right)$. If the difference between $\pi(\boldsymbol{d})$ and $\pi\left(\boldsymbol{d}^{\prime \prime}\right)$ are in two columns that are not adjacent, we can find another element between $\pi(\mathbf{d})$ and $\pi\left(\boldsymbol{d}^{\prime \prime}\right)$ by exchanging the order in which the two degrees are increased. This can also be done if the columns are adjacent, but the degrees differ by more than one.

If one of the two differ from $\pi\left(\boldsymbol{d}^{\prime}\right)$ in codimension and the other by an increase of the degree in one column, we can alter the order and obtain another element between $\pi(\boldsymbol{d})$ and $\pi\left(\boldsymbol{d}^{\prime \prime}\right)$.

The remaining cases are those described by (ii), (iii), and (iv).

Remark 3.6. In Example 3.4 matrices 1 and 12 correspond to boundary facets of type (i). Matrices 2 and 11 correspond to boundary facets of type (ii). Matrices 4 and 6 correspond to boundary facets of type (iii) and matrices 8 and 9 correspond 
to boundary facets of type (iv). The remaining matrices correspond to inner faces of the fan.

Theorem 3.7. The simplicial fan of pure diagrams in $B_{M, N}^{s}$ is convex.

Proof. We will use the following observation which allows us to go from local convexity to global convexity: If the simplicial fan is not convex, then there will be one boundary facet whose supporting hyperplane passes through the interior of a neighboring simplicial cone. We can see this by looking at a line segment between two vertices which contains points outside the simplicial fan. If we take a two-dimensional plane through these two points and through a generic inner point of the fan, we get a two-dimensional picture where we can find two edges meeting at an inwards angle. The supporting hyperplane of the boundary facet meeting the two-dimensional plane in one of these edges meets the interior of the simplicial cone corresponding to the other edge.

Thus we will prove that any two boundary facets meet in a convex manner. If either of the two facets are of type (i) or (ii), described in Proposition 3.5, it is clear that any Betti diagram will lie on the correct side of the supporting hyperplane, since this hyperplane is given by the vanishing of a single Betti number. Thus we will assume that the facets are of types (iii) or (iv).

Let $K$ denote the number of pure diagrams in a maximal chain in $B_{M, N}^{s}$. Any two boundary facets meeting along a codimension-one face gives us a chain with $K-2$ pure diagrams. If the two missing vertices are on levels differing by more than one, there is a unique way of completing the chain into a maximal chain and the two facets are faces of the same simplicial cone. Hence they meet in a convex way.

Thus we can assume that the two vertices missing are on adjacent levels and that there is a least element $\pi_{3}$ above these and a greatest element $\pi_{0}$ below them. Now we can see from the difference in codimension between $\pi_{0}$ and $\pi_{3}$ that facets of type (iii) described in Proposition 3.5 cannot meet facets of type (iv) in this way.

We must have that the two facets are given by removing $\pi_{1}^{\prime}$ or $\pi_{2}^{\prime \prime}$ from the chains $\pi_{0}<\pi_{1}^{\prime}<\pi_{2}^{\prime}<\pi_{3}$ and $\pi_{0}<\pi_{1}^{\prime \prime}<\pi_{2}^{\prime \prime}<\pi_{3}$ in the following lattice:

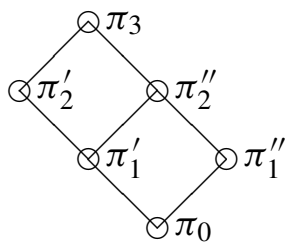

We need to show that the coefficient of $\pi_{1}^{\prime}$ is positive when $\pi_{1}^{\prime \prime}$ is expanded into the chain $\pi_{0}<\pi_{1}^{\prime}<\pi_{2}^{\prime}<\pi_{3}$. Note that the codimension cannot differ by more than three between $\pi_{0}$ and $\pi_{3}$ and if it differs by three, there is only one chain between $\pi_{0}$ and $\pi_{3}$. Thus we can assume that the difference in codimension is zero, one, or two. 
In order to do this we use Proposition 3.2 which gives us an expression of this coefficient involving only two terms.

Suppose that $\pi_{0}$ and $\pi_{3}$ differ in codimension by two. Then the coefficient is given by the fourth expression of Proposition 3.2 and the coefficient for $\pi_{1}^{\prime}=$ $\pi\left(d_{0}, d_{1}, \ldots, d_{m}\right)$ when $\pi_{1}^{\prime \prime}=\pi\left(d_{0}, d_{1}, \ldots, d_{k-1}, d_{k}+1, d_{k+1}, \ldots, d_{m}\right)$ is expanded in the basis containing $\pi_{0}<\pi_{1}^{\prime}<\pi_{2}^{\prime}$ is given by

$$
\sum_{i=0}^{n} \sum_{d \leq d_{i}\left(\pi_{0}\right)}(-1)^{i} \prod_{j=0}^{m-1}\left(d_{j}-d\right) \beta_{i, d}\left(\pi_{1}^{\prime \prime}\right)
$$

which has only two nonzero terms in columns $m$ and $m+1$ and equals

$$
\begin{gathered}
(-1)^{m} \prod_{j=0}^{m-1}\left(d_{j}-d_{m}\right)(-1)^{m} \frac{d_{k}-d_{m}}{d_{k}+1-d_{m}} \frac{1}{d_{m+1}-d_{m}} \prod_{j=0}^{m-1} \frac{1}{d_{j}-d_{m}} \\
+(-1)^{m+1} \prod_{j=0}^{m-1}\left(d_{j}-d_{m+1}\right)(-1)^{m+1} \frac{d_{k}-d_{m+1}}{d_{k}+1-d_{m+1}} \frac{1}{d_{m}-d_{m+1}} \prod_{j=0}^{m-1} \frac{1}{d_{j}-d_{m+1}} \\
=\frac{1}{d_{m+1}-d_{m}}\left(\frac{d_{k}-d_{m}}{d_{k}+1-d_{m}}-\frac{d_{k}-d_{m+1}}{d_{k}+1-d_{m+1}}\right) \\
=\frac{1}{\left(d_{k}+1-d_{m+1}\right)\left(d_{k}+1-d_{m}\right)}>0,
\end{gathered}
$$

since $d_{k}+1<d_{m}<d_{m+1}$.

If $\pi_{0}$ and $\pi_{3}$ differ in codimension by one we get that the coefficient of $\pi_{1}^{\prime}$ when $\pi_{1}^{\prime \prime}=\pi\left(d_{0}, d_{1}, \ldots, d_{m-1}\right)$ is expanded in the basis $\pi_{0}<\pi_{1}^{\prime}<\pi_{2}^{\prime}<\pi_{3}$ is given by

$$
\sum_{i=0}^{n} \sum_{d \leq d_{i}\left(\pi_{0}\right)}(-1)^{i}\left(d_{k}+2-d_{k}\right) \prod_{\substack{j=0 \\ j \notin\{k, k+1\}}}^{m}\left(d_{j}-d\right) \beta_{i, d}\left(\pi_{1}^{\prime \prime}\right),
$$

which has only two nonzero terms from columns $k$ and $k+1$ and equals

$$
\begin{aligned}
& 2(-1)^{k} \prod_{\substack{j=0 \\
j \notin\{k, k+1\}}}^{m}\left(d_{j}-d_{k}\right)(-1)^{k} \prod_{\substack{j=0 \\
j \neq k}}^{m-1} \frac{1}{d_{j}-d_{k}} \\
&+2(-1)^{k+1} \prod_{\substack{j=0 \\
j \notin k, k+1\}}}^{m}\left(d_{j}-d_{k}-1\right)(-1)^{k+1} \prod_{\substack{j=0 \\
j \neq k+1}}^{m-1} \frac{1}{d_{j}-d_{k}-1} \\
& \quad=2 \frac{d_{m}-d_{k}}{d_{k}+1-d_{k}}-2 \frac{d_{m}-d_{k}-1}{d_{k}-d_{k}-1}=2>0 .
\end{aligned}
$$


The last possibility is that the codimension of $\pi_{0}$ equals the codimension of $\pi_{3}$. In this case $\pi_{0}<\pi_{1}^{\prime}<\pi_{2}^{\prime}$ corresponds to a facet of type (iii) and we get that the coefficient of $\pi_{1}^{\prime}=\pi\left(d_{0}, d_{1}, \ldots, d_{k}, d_{k}+2, d_{k+2}, \ldots, d_{m}\right)$, when $\pi_{1}^{\prime \prime}=\pi\left(d_{0}, d_{1}\right.$, $\left.\ldots, d_{\ell-1}, d_{\ell}+1, d_{\ell+1}, \ldots, d_{m}\right)$ is expanded in the basis $\pi_{0}<\pi_{1}^{\prime}<\pi_{2}^{\prime}<\pi_{3}$, is given by

$$
\sum_{i=0}^{n} \sum_{d \leq d_{i}\left(\pi_{0}\right)}(-1)^{i}\left(d_{k}+2-d_{k}\right) \prod_{\substack{j=0 \\ j \notin\{k, k+1\}}}^{m}\left(d_{j}-d\right) \beta_{i, d}\left(\pi_{1}^{\prime \prime}\right) .
$$

Again there are only two nonzero terms and the coefficient is equal to

$$
\begin{aligned}
2(-1)^{k} \prod_{\substack{j=0 \\
j \notin\{k, k+1\}}}^{m}\left(d_{j}-d_{k}\right)(-1)^{k} \frac{d_{\ell}-d_{k}}{d_{\ell}+1-d_{k}} \prod_{\substack{j=0 \\
j \neq k}}^{m} \frac{1}{d_{j}-d_{k}} \\
+2(-1)^{k+1} \prod_{\substack{j=0 \\
j \notin\{k, k+1\}}}^{m}\left(d_{j}-d_{k}-1\right)(-1)^{k+1} \frac{d_{\ell}-d_{k}-1}{d_{\ell}+1-d_{k}-1} \prod_{\substack{j=0 \\
j \neq k+1}}^{m} \frac{1}{d_{j}-d_{k}-1} \\
\quad=2 \frac{d_{\ell}-d_{k}}{d_{\ell}+1-d_{k}}-2 \frac{d_{\ell}-d_{k}-1}{d_{\ell}-d_{k}}=\frac{2}{\left(d_{\ell}-d_{k}\right)\left(d_{\ell}-d_{k}+1\right)}>0,
\end{aligned}
$$

since $d_{\ell}-d_{k}$ and $d_{\ell}-d_{k}+1$ are both negative or both positive.

\section{The expansion of any Betti diagram into sums of pure diagrams}

We know from the work of Eisenbud and Schreyer that the inequalities given by the exterior facets of the cone are valid on all minimal free resolutions, not only on the resolutions of Cohen-Macaulay modules. The inequalities that we have to add because we now look at chains of pure diagrams of different codimensions can be seen to be limits of the inequalities already known.

As in the previous section, we look at Betti diagrams of graded modules under the restriction that $\beta_{i, j}=0$ unless $M+i \leq j \leq N+i$, that is, diagrams in $B_{M, N}$.

Theorem 4.1. Any Betti diagram of a finitely generated graded module $P$ of codimension s can be uniquely written as a positive linear combination of a chain of pure diagrams in $B_{M, N}^{s}$, where $M$ is the least degree of any generator of $P$ and $N$ is the regularity of $P$.

Proof. By Proposition 3.5 we know what are the boundary facets of the simplicial fan given by the pure diagrams in $B_{M, N}^{s}$ and by Proposition 3.2 we know how to obtain the inequalities given by the boundary facets. In fact, the coefficient of the removed element of the chain is nonnegative on the half-space defined by the corresponding boundary facet. We need to show that any Betti diagram of a graded module satisfies these inequalities. 
By Proposition 3.5 we have four types of boundary facets to consider, (i)-(iv). The inequalities corresponding to facets of type (i) and (ii) certainly hold for all Betti diagrams of modules.

The inequalities corresponding to facets of type (iii) are given by the expression of Proposition 3.2(c) and can be written as

$$
\sum_{i=0}^{n} \sum_{d=M}^{d_{i}\left(\pi_{0}\right)}(-1)^{i} \prod_{\substack{j=0 \\ j \notin\{k, k+1\}}}^{m}\left(d_{j}-d\right) \beta_{i, d}(P) \geq 0
$$

and the inequalities corresponding to facets of type (iv) are given by the expression of Proposition 3.2(d) and can be written as

$$
\sum_{i=0}^{n} \sum_{d=M}^{d_{i}\left(\pi_{0}\right)}(-1)^{i} \prod_{j=0}^{m-1}\left(d_{j}-d\right) \beta_{i, d}(P) \geq 0 .
$$

As we can see from these expressions, they are very similar. Indeed, (4-2) is equal to (4-1) if we increase $N$ and $m$ by one and choose $k=m-1$. Thus it is sufficient to prove that (4-1) always holds for any $m \leq n$.

The case $m=n$ corresponds to the facets that occur in the cone of CohenMacaulay diagrams. In the Cohen-Macaulay case we have more Herzog-Kühl equations but fewer inequalities. Eisenbud and Schreyer [2009, Theorems 4.1 and 7.1] proved that the inequalities given by the boundary facets hold for Betti diagrams of all modules, not only Cohen-Macaulay modules. Hence (4-1) holds for Betti diagrams in $B_{M, N}$ when $m=n$.

In order to prove that (4-1) holds when $m<n$, we look at the inequality we get by exchanging $\pi_{1}=\pi\left(d_{0}, d_{1}, \ldots, d_{m}\right)$ by $\pi_{1}^{t}=\pi\left(d_{0}, d_{1}, \ldots, d_{m}, d_{m}+1+t, d_{m}+\right.$ $\left.2+t, \ldots, d_{m}+n-m+t\right)$, and similarly exchanging $\pi_{0}$ and $\pi_{2}$ by $\pi_{0}^{t}$ and $\pi_{2}^{t}$. We then let $t$ grow to infinity and get

$$
\begin{aligned}
\lim _{t \rightarrow \infty} \frac{1}{t^{n-m}} \sum_{i=0}^{n} \sum_{d=M}^{d_{i}\left(\pi_{0}^{t}\right)}(-1)^{i} \prod_{\substack{j=0 \\
j \notin\{k, k+1\}}}^{m}\left(d_{j}-d\right) \prod_{j=m+1}^{n}\left(d_{m}+j-m+t-d\right) \beta_{i, d} \\
=\sum_{i=0}^{n} \sum_{d=M}^{d_{i}\left(\pi_{0}\right)}(-1)^{i} \prod_{\substack{j=0 \\
j \notin\{k, k+1\}}}^{m}\left(d_{j}-d\right) \beta_{i, d}(P) .
\end{aligned}
$$

The limit is nonnegative since for each integer $t \geq 0$, the expression under the limit in the left-hand side is nonnegative.

Remark 4.2. One of the questions raised by Eisenbud and Schreyer was what was the description of the convex cone cut out by all of their inequalities. The answer 
to this question is that this convex cone in $B_{M, N}$ equals the convex cone of all Betti diagrams in $B_{M, N}$ of graded modules up to multiplication by nonnegative rational numbers. This follows from Theorem 4.1 and the fact that all the inequalities we use to define the simplicial fan, as seen in the proof, are limits of their inequalities, hence are consequences of the full set of those inequalities. Thus for a given diagram satisfying their inequalities, we find suitable $M$ and $N$ and use Theorem 4.1 to see that it can be written as a positive linear combination of pure diagrams and we can conclude that a multiple of the diagram is the Betti diagram of a module.

We can also see that the unique way of writing any Betti diagram of a module into a chain of pure diagrams leads to a way of writing the diagram as a linear combination of diagrams of Cohen-Macaulay modules, one of each codimension between the codimension of $M$ and the projective dimension of $M$. As in the Cohen-Macaulay case, we get an algorithm for finding the expansion of a given Betti diagram by subtracting as much as possible of the pure diagram corresponding to the lowest shifts.

Remark 4.3. The method used in the proof of Theorem 4.1 is based only on what was known after the appearance of [Eisenbud and Schreyer 2009]. In view of the their more recent paper [Eisenbud and Schreyer 2010], the limiting process can be avoided using the functionals associated to coherent sheaves and, in fact, we only need to use vector bundles with supernatural cohomology supported on linear subspaces of $\mathbb{P}^{n-1}$, as pointed out by an anonymous referee.

Example 4.4. For $M=k[x, y, z] /\left(x^{2}, x y, x z^{2}\right)$ we get the Betti diagram

\begin{tabular}{c|cccc} 
& 1 & 3 & 3 & 1 \\
\hline $0:$ & 1 & - & - & - \\
$1:$ & - & 2 & 1 & - \\
$2:$ & - & 1 & 2 & 1
\end{tabular}

which can be expanded into

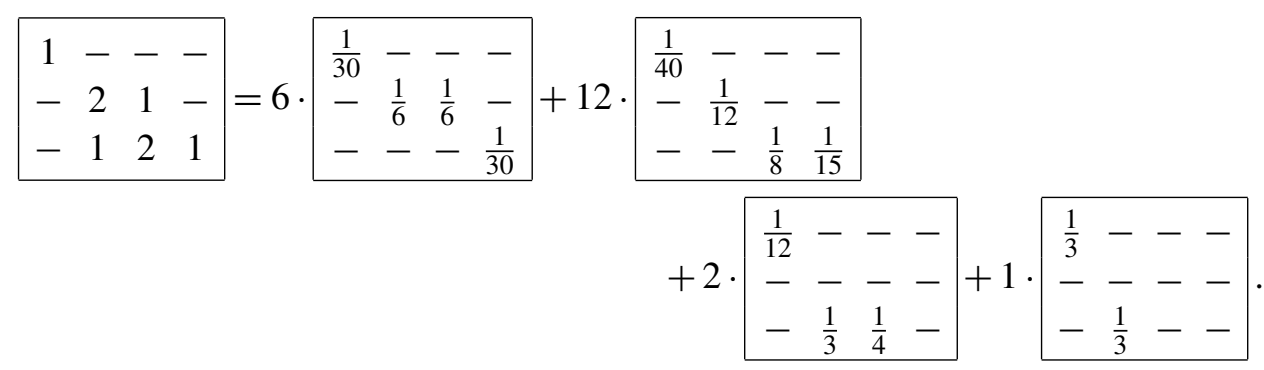

The coefficients can also be obtained by the functionals corresponding to the matrices 5, 6, 8, and 9 from Example 3.4. 
We now will go on and prove a generalized version of the multiplicity conjecture in terms of the Hilbert series. In order to do this, we first prove that the Hilbert series behaves well with respect to the partial ordering on the normalized pure diagrams. We use the partial order on Hilbert series given by coefficientwise inequality in all degrees simultaneously.

Proposition 4.5. The Hilbert series is strictly increasing on the partially ordered set of normalized pure diagrams generated in degree zero.

Proof. First assume that $\bar{\pi}<\bar{\pi}^{\prime}$ is a maximal chain of pure diagrams of codimension $s$. The Hilbert series can be recovered from the Betti diagrams and we can write

$$
H\left(\bar{\pi}^{\prime}, t\right)-H(\bar{\pi}, t)=\frac{1}{(1-t)^{n}}\left(S\left(\bar{\pi}^{\prime}, t\right)-S(\bar{\pi}, t)\right),
$$

where $S(\beta, t)=\sum_{i=0}^{n}(-1)^{i} \sum_{j} \beta_{i, j} t^{j}$. Since the polynomials $S(\bar{\pi}, t)$ and $S\left(\bar{\pi}^{\prime}, t\right)$ both have constant term 1 , we get that the polynomial $S\left(\bar{\pi}^{\prime}, t\right)-S(\bar{\pi}, t)$, has only $s+1$ nonzero terms. Since it also satisfies the Herzog-Kühl equations, we have a unique solution to this up to a scalar multiple, and $H(\bar{\pi}, t)-H\left(\bar{\pi}^{\prime}, t\right)$ is $\lambda t^{d_{1}}$ times the Hilbert series of a pure module. Since we know that all pure diagrams have positive Hilbert series, we only have to check that this is a nonnegative multiple. The sign of $\lambda$ can be obtained by looking at the sign of the term of $S\left(\bar{\pi}^{\prime}, t\right)-S(\bar{\pi}, t)$ which comes from $S\left(\bar{\pi}^{\prime}, t\right)$ and which is not present in $S(\bar{\pi}, t)$. This term comes with the sign $(-1)^{i}$, if it is in column $i$, which proves that $\lambda$ has to be positive.

We now consider the case where $\bar{\pi}<\bar{\pi}^{\prime}$ is a maximal chain such that the codimension of $\bar{\pi}$ is $s$ and the codimension of $\bar{\pi}^{\prime}$ is $s-1$. If we now look at the difference of the Hilbert series,

$$
H\left(\bar{\pi}^{\prime}, t\right)-H(\bar{\pi}, t)=\frac{1}{(1-t)^{n}}\left(S\left(\bar{\pi}^{\prime}, t\right)-S(\bar{\pi}, t)\right),
$$

where the polynomial $S\left(\bar{\pi}^{\prime}, t\right)-S(\bar{\pi}, t)$ has zero constant term and $s$ nonzero terms. Since we know that it is divisible by $(1-t)^{s-1}$, there is again a unique possibility, which is $\lambda t^{d_{1}}$ times the Hilbert series of a module with pure resolution. This time, we can see that the sign of the last term is $-(-1)^{s}=(-1)^{s-1}$, which shows that $\lambda$ is positive.

Theorem 4.6. For any finitely generated module $M$ of projective dimension $p$ and codimension $s$ generated in degree 0 , we have that

$$
H\left(\bar{\pi}\left(0, \underline{d}_{1}, \underline{d}_{2}, \ldots, \underline{d}_{p}\right), t\right) \leq \frac{H(M, t)}{\beta_{0}(M)} \leq H\left(\bar{\pi}\left(0, \bar{d}_{1}, \bar{d}_{2}, \ldots, \bar{d}_{s}\right), t\right),
$$

where $\underline{d}_{1}, \underline{d}_{2}, \ldots, \underline{d}_{p}$ are the minimal shifts and $\bar{d}_{1}, \bar{d}_{2}, \ldots, \bar{d}_{s}$ are the maximal shifts in a minimal free resolution of $M$. Equality on either side implies that the resolution is pure. 
In particular, the right-hand inequality implies the multiplicity conjecture, that is,

$$
\frac{e(M)}{\beta_{0}(M)} \leq \frac{\bar{d}_{1} \bar{d}_{2} \cdots \bar{d}_{s}}{s !}
$$

with equality if and only if $M$ is Cohen-Macaulay with a pure resolution.

Proof. We can use Theorem 4.1 to write the Betti diagram of $M$ as a positive linear combination of pure diagrams. Since the Hilbert series by Proposition 4.5 is increasing on along the chain, we get the first inequalities of the theorem.

The multiplicity of $M$ is obtained from the leading coefficient of the Hilbert polynomial. The coefficients of the Hilbert series are eventually equal to the Hilbert polynomial, which shows that the multiplicity is increasing with the Hilbert series as long as the degree of the Hilbert polynomial is the same. Since this is the case for $M$ and $\pi\left(0, \bar{d}_{1}, \bar{d}_{2}, \ldots, \bar{d}_{s}\right)$, we get the conclusion of the multiplicity conjecture.

Remark 4.7. For a Cohen-Macaulay module $M$, the Hilbert coefficients are positive linear combinations of the entries of the $h$-vector. Since we can reduce $M$ modulo a regular sequence and keep the same Betti diagram, we get that the Hilbert coefficients are increasing along chains of normalized pure diagrams. Thus the generalization of the multiplicity conjecture made by J. Herzog and X. Zheng [Herzog and Zheng 2009] is a consequence of Theorem 4.6.

\section{Acknowledgement}

This paper is heavily dependent on the beautiful proofs of our conjectures made by David Eisenbud and Frank-Olaf Schreyer and we are grateful for their efforts in this area. We would like to thank the participants at the workshop at Cornell University in March 2008 and the referee for useful comments and suggestions.

\section{References}

[Boij and Söderberg 2008] M. Boij and J. Söderberg, "Graded Betti numbers of Cohen-Macaulay modules and the multiplicity conjecture", J. Lond. Math. Soc.,(2) 78:1 (2008), 85-106. MR 2009g: 13018 Zbl 1189.13008

[Eisenbud and Schreyer 2009] D. Eisenbud and F.-O. Schreyer, "Betti numbers of graded modules and cohomology of vector bundles", J. Amer. Math. Soc. 22:3 (2009), 859-888. MR 2011a:13024 Zbl 1213.13032

[Eisenbud and Schreyer 2010] D. Eisenbud and F.-O. Schreyer, "Cohomology of coherent sheaves and series of supernatural bundles", J. Eur. Math. Soc. 12:3 (2010), 703-722. MR 2011e:14036 Zbl 1213.14035

[Eisenbud et al. 2011] D. Eisenbud, G. Fløystad, and J. Weyman, "The existence of equivariant pure free resolutions”, Ann. Inst. Fourier (Grenoble) 61:3 (2011), 905-926. MR 2918721 Zbl 139.13023

[Francisco 2006] C. A. Francisco, "New approaches to bounding the multiplicity of an ideal", $J$. Algebra 299:1 (2006), 309-328. MR 2007b:13043 Zbl 1105.13003 
[Francisco and Srinivasan 2007] C. A. Francisco and H. Srinivasan, "Multiplicity conjectures", pp. 145-178 in Syzygies and Hilbert functions, edited by I. Peeva, Lect. Notes Pure Appl. Math. 254, Chapman \& Hall/CRC, Boca Raton, FL, 2007. MR 2008f:13044 Zbl 1125.13011

[Francisco and Van Tuyl 2007] C. A. Francisco and A. Van Tuyl, "Some families of componentwise linear monomial ideals”, Nagoya Math. J. 187 (2007), 115-156. MR 2008g:13017 Zbl 1140.13012

[Goff 2008] M. Goff, "On the multiplicity conjecture for non-Cohen-Macaulay simplicial complexes”, preprint, 2008. arXiv 0802.1282v1

[Gold 2003] L. H. Gold, "A degree bound for codimension two lattice ideals", J. Pure Appl. Algebra 182:2-3 (2003), 201-207. MR 2004i:13023 Zbl 1032.13006

[Gold et al. 2007] L. Gold, H. Schenck, and H. Srinivasan, "Betti numbers and degree bounds for some linked zero-schemes", J. Pure Appl. Algebra 210:2 (2007), 481-491. MR 2008b:13020 Zbl 1123.14029

[Grayson and Stillman 1993] D. Grayson and M. Stillman, "Macaulay 2, a software system for research in algebraic geometry", 1993, Available at http://www.math.uiuc.edu/Macaulay2/.

[Guardo and Van Tuyl 2005] E. Guardo and A. Van Tuyl, "Powers of complete intersections: graded Betti numbers and applications", Illinois J. Math. 49:1 (2005), 265-279. MR 2006k:13035 Zbl 1089.13008

[Herzog and Kühl 1984] J. Herzog and M. Kühl, "On the Betti numbers of finite pure and linear resolutions”, Comm. Algebra 12:13-14 (1984), 1627-1646. MR 85e:13021 Zbl 0543.13008

[Herzog and Srinivasan 1998] J. Herzog and H. Srinivasan, "Bounds for multiplicities", Trans. Amer. Math. Soc. 350:7 (1998), 2879-2902. MR 99g:13033 Zbl 0899.13026

[Herzog and Zheng 2006] J. Herzog and X. Zheng, "Notes on the multiplicity conjecture", Collect. Math. 57:2 (2006), 211-226. MR 2007a:13029 Zbl 1116.13022

[Herzog and Zheng 2009] J. Herzog and X. Zheng, "Bounds for Hilbert coefficients", Proc. Amer. Math. Soc. 137:2 (2009), 487-494. MR 2009f:13024 Zbl 1162.13010

[Hibi and Singla 2008] T. Hibi and P. Singla, "Linear balls and the multiplicity conjecture", J. Algebra 319:10 (2008), 4372-4390. MR 2009e:13026 Zbl 1166.13026

[Huneke and Miller 1985] C. Huneke and M. Miller, "A note on the multiplicity of Cohen-Macaulay algebras with pure resolutions", Canad. J. Math. 37:6 (1985), 1149-1162. MR 87d:13024 Zbl 0579. 13012

[Kubitzke and Welker 2006] M. Kubitzke and V. Welker, "The multiplicity conjecture for barycentric subdivisions", preprint, 2006. arXiv math/0606274v1

[Migliore et al. 2005] J. Migliore, U. Nagel, and T. Römer, "The multiplicity conjecture in low codimensions”, Math. Res. Lett. 12:5 (2005), 731-747. MR 2006i:13042 Zbl 1090.13020

[Migliore et al. 2008a] J. Migliore, U. Nagel, and T. Römer, "Extensions of the multiplicity conjecture”, Trans. Amer. Math. Soc. 360:6 (2008), 2965-2985. MR 2009a:13043 Zbl 1148.13016

[Migliore et al. 2008b] J. C. Migliore, U. Nagel, and F. Zanello, "An improved multiplicity conjecture for codimension 3 Gorenstein algebras”, Comm. Algebra 36:1 (2008), 112-119. MR 2008k:13033 Zbl 1135.13012

[Miró-Roig 2006] R. M. Miró-Roig, "A note on the multiplicity of determinantal ideals", J. Algebra 299:2 (2006), 714-724. MR 2007a:13030 Zbl 1116.13008

[Puthenpurakal 2008] T. J. Puthenpurakal, "On the upper bound of the multiplicity conjecture", Proc. Amer. Math. Soc. 136:10 (2008), 3429-3434. MR 2009e:13029 Zbl 1163.13018

[Römer 2005] T. Römer, "Note on bounds for multiplicities", J. Pure Appl. Algebra 195:1 (2005), 113-123. MR 2005g:13041 Zbl 1073.13015 
[Seo and Srinivasan 2007] S. Seo and H. Srinivasan, "Multiplicity of codimension three almost complete intersections", preprint, 2007. arXiv arXiv:0712.0818v1

[Srinivasan 1998] H. Srinivasan, "A note on the multiplicities of Gorenstein algebras", J. Algebra 208:2 (1998), 425-443. MR 99m:13046 Zbl 0920.13018

[Zanello 2007] F. Zanello, "Improving the bounds of the multiplicity conjecture: the codimension 3 level case”, J. Pure Appl. Algebra 209:1 (2007), 79-89. MR 2008a:13034 Zbl 1116.13023

Communicated by David Eisenbud

Received 2010-07-02 Revised 2011-01-24 Accepted 2011-05-23

boij@kth.se

Department of Mathematics, KTH, SE-100 44 Stockholm, Sweden

jonassod@gmail.com

Department of Mathematics, KTH, SE-100 44 Stockholm, Sweden 


\section{Algebra \& Number Theory}

msp.berkeley.edu/ant

\section{EDITORS}

MANAGING EDITOR

Bjorn Poonen

Massachusetts Institute of Technology

Cambridge, USA

\author{
EDITORIAL BOARD CHAIR \\ David Eisenbud \\ University of California \\ Berkeley, USA
}

\section{BOARD OF EDITORS}

Georgia Benkart

Dave Benson

Richard E. Borcherds

John H. Coates

J-L. Colliot-Thélène

Brian D. Conrad

Hélène Esnault

Hubert Flenner

Edward Frenkel

Andrew Granville

Joseph Gubeladze

Ehud Hrushovski

Craig Huneke

Mikhail Kapranov

Yujiro Kawamata

János Kollár

Yuri Manin

Barry Mazur

Philippe Michel

Susan Montgomery
University of Wisconsin, Madison, USA

University of Aberdeen, Scotland

University of California, Berkeley, USA

University of Cambridge, UK

CNRS, Université Paris-Sud, France

University of Michigan, USA

Universität Duisburg-Essen, Germany

Ruhr-Universität, Germany

University of California, Berkeley, USA

Université de Montréal, Canada

San Francisco State University, USA

Hebrew University, Israel

University of Kansas, USA

Yale University, USA

University of Tokyo, Japan

Princeton University, USA

Northwestern University, USA

Harvard University, USA

École Polytechnique Fédérale de Lausanne

University of Southern California, USA
Shigefumi Mori

Raman Parimala

Jonathan Pila

Victor Reiner

Karl Rubin

Peter Sarnak

Joseph H. Silverman

Michael Singer

Ronald Solomon

Vasudevan Srinivas

J. Toby Stafford

Bernd Sturmfels

Richard Taylor

Ravi Vakil

Michel van den Bergh

Marie-France Vignéras

Kei-Ichi Watanabe

Andrei Zelevinsky

Efim Zelmanov
RIMS, Kyoto University, Japan

Emory University, USA

University of Oxford, UK

University of Minnesota, USA

University of California, Irvine, USA

Princeton University, USA

Brown University, USA

North Carolina State University, USA

Ohio State University, USA

Tata Inst. of Fund. Research, India

University of Michigan, USA

University of California, Berkeley, USA

Harvard University, USA

Stanford University, USA

Hasselt University, Belgium

Université Paris VII, France

Nihon University, Japan

Northeastern University, USA

University of California, San Diego, USA

\section{PRODUCTION}

contact@msp.org

Silvio Levy, Scientific Editor

See inside back cover or www.jant.org for submission instructions.

The subscription price for 2012 is US \$175/year for the electronic version, and \$275/year (+\$40 shipping outside the US) for print and electronic. Subscriptions, requests for back issues from the last three years and changes of subscribers address should be sent to Mathematical Sciences Publishers, Department of Mathematics, University of California, Berkeley, CA 94720-3840, USA.

Algebra \& Number Theory (ISSN 1937-0652) at Mathematical Sciences Publishers, Department of Mathematics, University of California, Berkeley, CA 94720-3840 is published continuously online. Periodical rate postage paid at Berkeley, CA 94704, and additional mailing offices.

ANT peer review and production are managed by EditFLOW ${ }^{\circledR}$ from Mathematical Sciences Publishers.

PUBLISHED BY

mathematical sciences publishers

http://msp.org/

A NON-PROFIT CORPORATION

Typeset in IAT $_{\mathrm{E}} \mathrm{X}$

Copyright (C2012 by Mathematical Sciences Publishers 


\section{Algebra \& Number Theory}

Volume $6 \quad$ No. $3 \quad 2012$

The image of complex conjugation in $l$-adic representations associated to automorphic forms

\section{RICHARD TAYLOR}

Betti numbers of graded modules and the multiplicity conjecture in the

non-Cohen-Macaulay case

\section{MATS BOIJ and JONAS SÖDERBERG}

$\mathscr{L}$-invariants and Shimura curves

SAMIT DAsGupta and MATTHEW GREENBERG

On the weak Lefschetz property for powers of linear forms

Juan C. Migliore, Rosa M. Miró-Roig and Uwe Nagel

Resonance equals reducibility for $A$-hypergeometric systems

Mathias Schulze and Uli WaLther

The Chow ring of double EPW sextics

ANDREA FERRETTI

A finiteness property of graded sequences of ideals

Mattias JONSSON and Mircea MustaȚĂ

On unit root formulas for toric exponential sums

Alan Adolphson and SteVen Sperber

Symmetries of the transfer operator for $\Gamma_{0}(N)$ and a character deformation of the 